\title{
Assessing alternatives for directional detection of a halo of weakly interacting massive particles
}

\author{
Craig J. Copi,${ }^{1, *}$ Lawrence M. Krauss,${ }^{1,2, \dagger}$ David Simmons-Duffin, ${ }^{3}$ and Steven R. Stroiney ${ }^{4}$ \\ ${ }^{1}$ Department of Physics, Center for Education and Research in Cosmology and Astrophysics, \\ 10900 Euclid Ave., Cleveland, Ohio 44106-7079, USA \\ ${ }^{2}$ Department of Astronomy, Case Western Reserve University, 10900 Euclid Ave., Cleveland, Ohio 44106-7079, USA \\ ${ }^{3}$ Physics Department, Harvard University, Cambridge, Massachusetts 02138, USA \\ ${ }^{4}$ Physics Department, Cornell University, Ithaca, New York 14853, USA
}

(Received 30 August 2005; published 12 January 2007)

\begin{abstract}
The future of direct terrestrial WIMP detection lies on two fronts: new, much larger low background detectors sensitive to energy deposition, and detectors with directional sensitivity. The former can explore a large range of WIMP parameter space using well-tested technology while the latter may be necessary if one is to disentangle particle physics parameters from astrophysical halo parameters. Because directional detectors will be quite difficult to construct it is worthwhile exploring in advance generally which experimental features will yield the greatest benefits at the lowest costs. We examine the sensitivity of directional detectors with varying angular tracking resolution with and without the ability to distinguish forward versus backward recoils, and compare these to the sensitivity of a detector where the track is projected onto a two-dimensional plane. The latter detector regardless of where it is placed on the Earth, can be oriented to produce a significantly better discrimination signal than a $3 \mathrm{D}$ detector without this capability, and with sensitivity within a factor of 2 of a full 3D tracking detector. Required event rates to distinguish signals from backgrounds for a simple isothermal halo range from the low teens in the best case to many thousands in the worst.
\end{abstract}

DOI: 10.1103/PhysRevD.75.023514

PACS numbers: 95.35.+d, 95.55. Vj

\section{INTRODUCTION}

Direct detection experiments for weakly interacting massive particles (WIMPs) continue to set ever more stringent limits on the nucleon-WIMP cross section [1-5]. A precise understanding of the backgrounds is required to identify an excess of nuclear recoils. There is no unique signature that can separate neutron induced recoils from WIMP induced recoils in these detectors. Even annual modulation, which is at best a few percent effect, might be accounted for by seasonal background variations. (This is for a pure WIMP signal. When a uniform background is included the effect is even smaller.) It has been recognized that a stronger signal comes from measuring the direction of the recoiling nucleus [6] allowing for a WIMP signal to be identified from a few events even in the presence of backgrounds $[7,8]$. Detectors that might measure the recoil direction have been designed and built. For example, time projection chambers have been used by DRIFT [9] and NEWAGE [10] and a scintillator with direction dependent response has also been studied [11].

In their present state the directional detectors are rudimentary at best. The current version of DRIFT, for example, does not measure the full three-dimensional track of the recoiling nucleus. Instead it measures the recoil track projected onto a plane and has a number of other limitations. A detailed statistical study of a 3 dimensional detector, such as DRIFT II, has been performed [12].

\footnotetext{
*Electronic address: cjc5@cwru.edu

†Electronic address: 1mk9@cwru.edu
}

A new generation of directionally sensitive detectors are now being envisaged. Building a full three-dimensional detector is a challenging, costly proposition. Is it necessary? Given the technical challenge and cost constraints of these detectors, which changes will lead to the most sensitive detector? Here we provide a general analysis of various design goals to determine the number of events required for detector designs ranging from twodimensional to fully three-dimensional detectors. We do not focus on any particular detector technology nor model existing or planned detectors. Instead we apply a consistent set of parameters to a variety of detector configurations. This allows us to determine the optimal design goals independent of detector details.

\section{THEORETICAL MODEL}

\section{A. Detector characteristics}

To quantify the capabilities of directionally sensitive detectors we consider a consistent, generic set of parameters. For the detector target we use a xenon $\left(m_{N}=\right.$ $131 \mathrm{GeV}$ ) nucleus. We assume a threshold of $Q_{\mathrm{th}}=$ $10 \mathrm{keV}$ and two different WIMP masses, $m_{\chi}=100 \mathrm{GeV}$ $\left(m_{\chi} \approx m_{N}\right)$ and $m_{\chi}=1000 \mathrm{GeV}\left(m_{\chi} \gg m_{N}\right)$. For our Galaxy we focus solely on an isothermal model for the WIMP halo distribution,

$$
f(\vec{v})=\frac{1}{\pi^{3 / 2} v_{0}^{3}} e^{-|\vec{v}|^{2} / v_{0}^{2}}
$$

We study three different values for $v_{0}$ spanning the range of current expectations, $170 \mathrm{~km} / \mathrm{s}, 220 \mathrm{~km} / \mathrm{s}$, and 
$270 \mathrm{~km} / \mathrm{s}$. The escape velocity of WIMPs from the Galaxy is taken to be $650 \mathrm{~km} / \mathrm{s}$. The Earth's rotation axis is oriented at an angle $\epsilon=42^{\circ}$ with respect to the Sun's motion. This value is relevant for the two-dimensional detector. We stress that these choices have been made to provide a consistent set of parameters to allow the intercomparison of detector designs not as a suggestion for an actual detector design. Thus, what will be important about our results will not be absolute constraints, but relative ones, although we expect the overall order of magnitude for the required event rates will not differ compared to our estimates.

\section{B. Differential event rates}

The technique for calculating the WIMP scattering rate is well known [7]. The differential rate as a function of nuclear recoil direction $(\gamma, \phi)$ is given by

$$
\frac{d R}{d \Omega_{\gamma, \phi}}=\frac{\sigma_{0} \rho_{0}}{\pi m_{N} m_{\chi}} \int_{\mathcal{R}} d^{3} \vec{v} J v F^{2}(Q(J v)) f\left(\vec{v}+\vec{v}_{\oplus}\right) .
$$

Here $m_{N}$ is the mass of the target nucleus, $m_{\chi}$ is the WIMP mass, $\vec{v}_{\oplus}$ is the velocity of the Earth through the WIMP halo, $Q$ is the recoil energy of the nucleus, $\sigma_{0}$ is the cross section for WIMP scattering off the target nucleus, and $\rho_{0}$ is the local WIMP halo density. We consider only spin independent interactions and use the standard Helm form factor [13] for $F^{2}(Q)$. The geometry of the WIMP scattering gives

$$
J v \equiv v_{x} \sin \gamma \cos \phi+v_{y} \sin \gamma \sin \phi+v_{z} \cos \gamma,
$$

which relates the direction of the incoming WIMP to the direction of the recoiling nucleus. The integration region, $\mathcal{R}$, is defined by the detector threshold, $Q_{\mathrm{th}}$, at the lower limit,

$$
J v \geq \sqrt{\frac{\left(m_{\chi}+m_{N}\right)^{2}}{2 m_{\chi}^{2} m_{N}} Q_{\mathrm{th}}},
$$

and the galactic escape velocity, $v_{\text {esc }}$, at the upper limit,

$$
\left(v_{x}+v_{\oplus, x}\right)^{2}+\left(v_{y}+v_{\oplus, y}\right)^{2}+\left(v_{z}+v_{\oplus, z}\right)^{2} \leq v_{\text {esc }}^{2} .
$$

See [7] for a more detailed discussion. A full threedimensional detector probes the reaction rate outlined here (2).

Measuring the direction the nucleus is moving along a track is not always possible. For a three-dimensional detector without forward-backward discrimination, a recoil in a direction $(\cos \gamma, \phi)$ cannot be distinguished from a recoil in a direction $(-\cos \gamma, \phi+\pi)$. The event rate for these two directions thus combine giving a total rate for the direction $(\cos \gamma, \phi)$ of $\frac{d R}{d \Omega}(\cos \gamma, \phi)+\frac{d R}{d \Omega}(-\cos \gamma, \phi+\pi)$ where the differential rates are again given above (2).

A two-dimensional detector can only resolve the recoil direction projected onto a plane. We assume for compari- son purposes however that it can be designed with forwardbackward discrimination. Suppose the normal to the detector plane is oriented at angles $(\zeta, \eta)$ with respect to the direction of the Sun's motion. The rate is a function of a single angle $\phi^{\prime}$ measured in this frame,

$$
\begin{aligned}
\frac{d R}{d \phi^{\prime}}= & \frac{\sigma_{0} \rho_{0}}{\pi m_{N} m_{\chi}} \int_{-1}^{1} d\left(\cos \gamma^{\prime}\right) \int_{\mathcal{R}} d^{3} \vec{v}^{\prime}(J v)^{\prime} \\
& \times F^{2}\left(Q^{\prime}\right) f\left(\vec{v}+\vec{v}_{\oplus}\right) .
\end{aligned}
$$

Here, primed coordinates are measured in the frame of the detector (where the normal vector points along the $z$-axis) and unprimed coordinates are measured in the frame of the Sun's motion. These two frames are related by rotations through the angles $\zeta$ and $\eta$.

For a detector fixed to the Earth's surface, the detector orientation with respect to the Sun's motion changes throughout the day due to the Earth's rotation. Let $\theta$ be the angle between the detector's normal and the Earth's rotation axis and $\epsilon$ be the angle between the rotation axis and the Sun's velocity. Then

$$
\cos \zeta=\cos \epsilon \cos \theta+\sin \epsilon \sin \theta \cos (2 \pi t)
$$

for $t$ measured in days.

It is also important to recognize that even full threedimensional detectors will not have perfect angular resolution. To model realistic angular resolution either due to dispersion of the recoiling nucleus along its ideal track or due to inherent precision of the inherent detection mechanism itself we convolve the ideal scattering rate (2) with a smoothing kernel $K\left(\Omega, \Omega^{\prime}\right)$,

$$
\frac{d R}{d \Omega}(\Omega)=\int K\left(\Omega, \Omega^{\prime}\right) \frac{d R}{d \Omega}\left(\Omega^{\prime}\right) d \Omega^{\prime} .
$$

We use a Gaussian smoothing about the direction of the ideal recoil,

$$
K\left(\Omega, \Omega^{\prime}\right)=e^{-\xi^{2} / 2 \sigma^{2}} /(2 \pi)^{3 / 2} \sigma \operatorname{erf}(\sqrt{2} \sigma),
$$

where

$$
\cos \xi=\sin \theta \sin \theta^{\prime} \cos \left(\phi-\phi^{\prime}\right)+\cos \theta \cos \theta^{\prime} .
$$

We study this as a function of the width of the Gaussian.

\section{RESULTS}

We test the capabilities of each type of WIMP detector by assessing their ability to distinguish the WIMP distribution from a flat background, using an isothermal halo as a fiducial test model. The probability that a WIMP will recoil in a particular direction, $\Omega_{i}$, is given by $P\left(\Omega_{i}\right)=$ $\frac{d R}{d \Omega}\left(\Omega_{i}\right)$, where we have normalized the rate such that $R=$ 1 . Thus, we are probing the shape of the recoil spectrum. The likelihood function for $N_{e}$ detected events is defined by $\mathcal{L}=\prod_{i=1}^{N_{e}} P\left(\Omega_{i}\right)$. We generate at least 100000 sample distributions for each $N_{e}$ and apply the log-likelihood test to find the minimum number of WIMP events such that we 
have a $95 \%$ detection $95 \%$ of the time (see [7] for more details).

The results for $m_{\chi}=100 \mathrm{GeV}$ are given in Table I for the range of detectors we have considered, where "full" reflects a full three-dimensional detector with perfect angular resolution. We shall discuss the degradation implied by limited resolution shortly. The same set of results for $m_{\chi}=1000 \mathrm{GeV}$ are given in Table II. Although we have restricted our quantitative study to isothermal models the qualitative features of the comparison remain valid for other models, including models with single streams of WIMPs, but with a significant isothermal component.

Our results underscore the need for forward-backward detection. Indeed, this is the single most important feature that allows directional detectors to gain sensitivity to the WIMP signal compared to backgrounds. Since spin independent WIMP scattering is azimuthally symmetric about the direction of the incoming WIMP the dominant WIMP signal comes from the a comparison of forward-backward scattering events. This is seen in the results in Tables I and II. A three-dimensional detector, even with perfect angular resolution, but without forward-backward discrimination requires a surprisingly large (at least an order of magnitude greater) number of events than a three-dimensional detector with such discrimination and even many more than a poorly aligned two-dimensional detector to distinguish a WIMP signal from terrestrial backgrounds. This is because without forward-backward discrimination the detector relies upon the difference between head-on and glancing (wide angle) collisions as well as high angular resolution to distinguish a WIMP signal from the background. The surprisingly large difference in required number of events

TABLE I. The number of events required to identify a WIMP signal above a flat background for different types of detectors and a WIMP mass of $m_{\chi}=100 \mathrm{GeV}$.

\begin{tabular}{lccc}
\hline \hline Detector Type & \multicolumn{3}{c}{$\boldsymbol{v}_{0}(\mathrm{~km} / \mathrm{s})$} \\
\hline 3D (full) & 170 & 220 & 270 \\
3D without FB & 6 & 11 & 18 \\
2D-best/worst & 176 & 1795 & $>35,000$ \\
2D rotating & $19 / 45$ & $34 / 75$ & $61 / 123$ \\
\hline \hline
\end{tabular}

TABLE II. Same as Table I, for a WIMP mass of $m_{\chi}=$ $1000 \mathrm{GeV}$.

\begin{tabular}{lccc}
\hline \hline Detector Type & \multicolumn{3}{c}{$\boldsymbol{v}_{0}(\mathrm{~km} / \mathrm{s})$} \\
\hline 3D (full) & 170 & 220 & 270 \\
3D without FB & 14 & 27 & 51 \\
2D fixed-best/worst & 152 & 217 & 371 \\
2D rotating & $51 / 129$ & $97 / 217$ & $175 / 368$ \\
\hline \hline
\end{tabular}

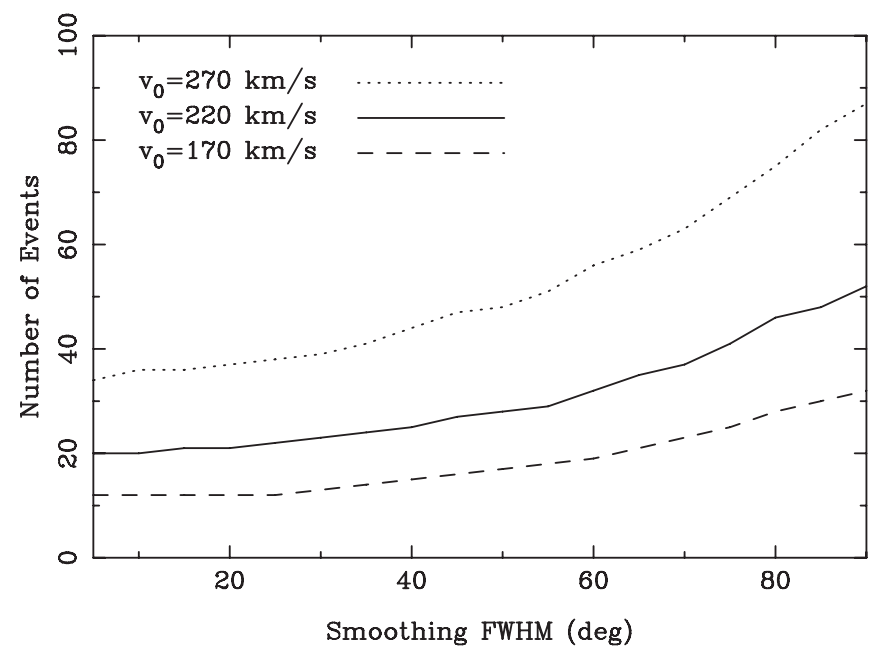

FIG. 1. The number of events required as a function of the fullwidth half maximum (FWHM) of the smoothing kernel (9) for the isothermal models, $m_{\chi}=100 \mathrm{GeV}$, and the detector configuration described in section II A.

for heavy WIMPs versus WIMPs of mass comparable to target nuclei masses presumably is due to the fact that nuclear recoils from collisions with heavy WIMPS tend to better follow the direction of the original WIMP.

We next explore how the sensitivity of a threedimensional detector depends upon its angular resolution. In Fig. 1 we display the number of events required as a function of the angle of the full-width half maximum (FWHM) of the detection cone for the events. Note that as the angular resolution degrades, the number of events required for a three-dimensional detector quickly approaches that of a two-dimensional detector, as expected. In order to be significantly more efficient, the angular

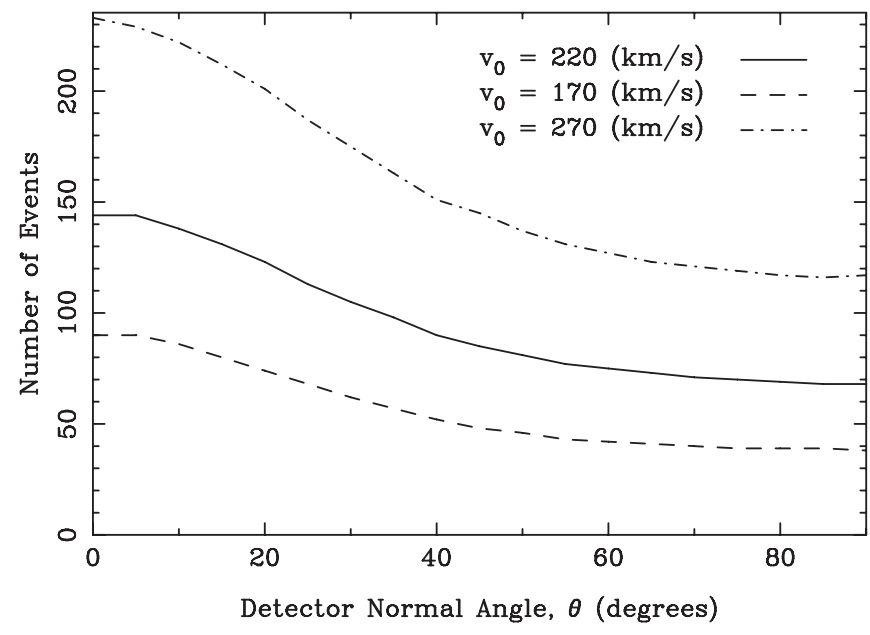

FIG. 2. The number of events required as a function of the angle between the detector normal and the direction of the WIMP wind (Sun's motion), $\theta$ for the isothermal models, $m_{\chi}=$ $100 \mathrm{GeV}$, and the detector configuration described in Sec. II A. 
resolution of such a detector must be better than about 60 degrees (FWHM).

We finally focus on two-dimensional detectors, in part because these are likely to be the most practical in the near future, and because less attention has been paid to them than hypothetical three-dimensional detectors.

It is clear that the efficacy of a planar detector will depend upon the orientation of its plane with respect to the direction of the WIMP wind. Specifically a twodimensional detector fixed to the Earth will be oriented so that its normal vector makes an angle $\theta$ with the Earth's rotation axis. The choice of $\theta$ determines how much time the detector will spend at various angles $\zeta$ relative to the Earth's direction of motion. An orientation of $\zeta=0^{\circ}$ is clearly the worst since the detector plane is then perpendicular to the WIMP wind. The number of events required is a function of the angle $\theta$ chosen for the detector as shown in Fig. 2.

Tables I and II give the minimum and maximum number of events required for optimal versus worst-case orientation of the detector. Note that the shape of the function in Fig. 2 depends on the orientation of the Earth's axis, $\epsilon=$ $42^{\circ}$ relative to the motion of the Sun through an isotropic halo. For halos in which a WIMP stream arose which was not due to the motion of the Sun (i.e. involving some other bulk motion with respect to the galaxy rest frame, as would occur for some infalling WIMP distribution), the shape of the curve would change. Low values of $\epsilon$ in this case would produce a more steeply descending function, and high values would produce an ascending function.

Note that the results thus far assume that the detector axis is fixed to the plane of the Earth. In this case the time averaged number of events required is on average 3 times less for a two-dimensional detector oriented in the best possible axis (i.e. at or near $\theta=90^{\circ}$ for isothermal halo distribution). Such a detector however requires three to four times the number of events of a full three-dimensional detector. Whether or not achieving this additional factor of 3 in sensitivity for a full three-dimensional detector is worth the technological challenge is not clear. However, as seen in the tables, this factor of 3 can be reduced by using a detector which is not fixed relative to the plane of the Earth, but which can rotate over the course of each day with respect to the earth to maintain an optimal orientation with respect to an expected WIMP wind. For an isothermal halo, roughly only 2 times as many events are required for such a rotating detector compared to a detector with full three-dimensional tracking capability. The technical difficult of producing the former may be less demanding than that required to produce the latter. Our purpose is to demonstrate the theoretical gain that may be obtained so experimentalists can then decide if the challenge is worth addressing.

Our results can be summarized as follows: For directional WIMP detectors, forward-backward discrimination is far more valuable than three-dimensional resolution of the track, at least for the isothermal model considered here. Furthermore, a two-dimensional detector can be oriented into the predominant WIMP wind so that the number of events required to distinguish a WIMP halo from a terrestrial background is comparable to that required for even a full three-dimensional detector. Note that the results for a two-dimensional detector are independent of its location on the Earth. The orientation of the plane of the detector determines its capabilities not the latitude of the detector. Some locations may allow for easier detector orientation $[14,15]$ but all locations are equally good provided the detector plane can be properly aligned. While there may be other reasons (including background rejection) for considering fully three-dimensional directional detection methods, our results thus suggest that concentrating simply on forward-background sensitivity is the most important new direction that should be pursued in directional WIMP detection, and that planar detectors can, in this case, provide nearly optimal directional sensitivity. These results are consistent with those obtained in a complimentary work that studied the specific capabilities of a DRIFTtype detector [14].
[1] R. Bernabei, P. Belli, R. Cerulli, F. Montecchia, M. Amato, G. Ignesti, A. Incicchitti, D. Prosperi, C. J. Dai, and H. L. He et al., Phys. Lett. B 480, 23 (2000).

[2] D. S. Akerib, J. Alvaro-Dean, M. S. Armel, M. J. Attisha, L. Baudis, D. A. Bauer, A. I. Bolozdynya, P. L. Brink, R. Bunker, and B. Cabrera et al., Phys. Rev. D 68, 082002 (2003).

[3] S. Marnieros, A. Benoit, L. Bergé, A. Broniatowski, B. Censier, L. Chabert, B. Chambon, M. Chapellier, G. Chardin, and P. Charvin et al., Nucl. Instrum. Methods Phys. Res., Sect. A 520, 101 (2004).
[4] J. C. Barton, G. J. Alner, G. J. Arnison, R. C. Carter, S. P. Hart, G. Homer, J. D. Lewin, R. Lüscher, R. M. Preece, and J.W. Roberts et al., in Proceedings of the Fourth International Workshop on the Identification of Dark Matter, edited by N.J.C. Spooner and V. Kudryavtsev (World Scientific, Singapore, 2003), pp. 302-307.

[5] D. S. Akerib et al. (CDMS), Phys. Rev. Lett. 93, 211301 (2004).

[6] D. N. Spergel, Phys. Rev. D 37, 1353 (1988).

[7] C. J. Copi, J. Heo, and L. M. Krauss, Phys. Lett. B 461, 43 (1999). 
[8] C. J. Copi and L. M. Krauss, Phys. Rev. D 63, 043507 (2001).

[9] D. P. Snowden-Ifft, C. J. Martoff, and J. M. Burwell, Phys. Rev. D 61, 101301 (2000).

[10] T. Tanimori, H. Kubo, K. Miuchi, T. Nagayoshi, R. Orito, A. Takada, and A. Takeda, Phys. Lett. B 578, 241 (2004).

[11] H. Sekiya, M. Minowa, Y. Shimizu, W. Suganuma, and Y. Inoue, astro-ph/0405598.
[12] B. Morgan, A. M. Green, and N. J. Spooner, Phys. Rev. D 71, 103507 (2005).

[13] R. H. Helm, Phys. Rev. 104, 1466 (1956).

[14] B. Morgan and A. M. Green, Phys. Rev. D 72, 123501 (2005).

[15] P. F. Smith et al. (UK Dark Matter), Phys. Rep. 307, 275 (1998). 